\title{
Proyección ambiental del eje verde del Rio Monquira para la mitigación de la contaminación en Sogamoso- Boyacá
}

\section{Environmental projection of the green axis of the Monquira River for the mitigation of pollution in Sogamoso -Boyacá}

\author{
Mario Fernando Sánchez Sierra ${ }^{1}$;; Karen Ximena Villamarin Caro ${ }^{2}$ \\ ${ }^{1}$ Investigador SA21 consultores, Arquitectura Universidad de Boyacá. \\ marfersanchez@uniboyaca.edu.co \\ 2 Investigador SA21 consultores, Urbanismo y medio ambiente, ximena21_08@hotmail.com
}

\section{Resumen}

Preservar las fuentes hídricas para el cambio climático es una necesidad obligada en los municipios. Esta necesidad nace de observar el deterioro constante del Rio y el abandono por parte de las autoridades locales. Este proyecto pretende realizar un diagnóstico actual de la situación del agua, de la rivera y de los usos del Rio, esto con el fin de poder proponer una intervención del Rio como eje verde para la ciudad que mitigue la alta contaminación que tiene Sogamoso por su industria, alfarería y comercio. El rio Monquira una herencia ambiental del cual debe desprender una cultura del agua, de movilidad alternativa, de apreciación de recursos, de la flora y fauna existente para la preservación de la misma. El trabajo se desarrolla partiendo de un diagnóstico para plantear un nuevo modelo de intervención urbana. Se usó una metodología proyectiva, para poder estudiar los elementos en su contexto Relaciones dinámicas, se interesa en los procesos evolutivos y las relaciones dinámicas entre los eventos Creatividad y participación son vitales en el proceso de construir una movilidad alternativas y unos espacios públicos que contribuyan.
Potencializar el eje verde como alternativa de desarrollo económico y ambiental que desde la investigación de soluciones para el ecosistema social y ambiental dentro de la ciudad para la rehabilitación del eje verde del "Rio Monquira" siendo esta una de nuestras fuentes hídricas más importantes, debido a que los cuerpos de agua en una ciudad son vitales frente a la reducción del $\mathrm{CO}$ y el mejoramiento del aire.

Palabras clave: cambio climático, eje verde, mitigación, Rio Monquira

\section{Abstract}

To preserve the water sources for the climate change is a need forced in the municipalities. This need is born of observing the constant deterioration of the Rio and the abandonment on the part of the local authorities. This project tries to realize a current diagnosis of the situation of the water, of the rivera and of the uses of the Rio, this in order to be able to propose an intervention of the Rio as green axis for the city that mitigates the high contamination that Sogamoso takes as its industry, pottery and commerce. The river Monquira an 
93

environmental heredity of which debit to detach a culture of the water, of alternative mobility, of appreciation of resources, of the flora and existing fauna for the preservation of the same one. The work develops departing from a diagnosis to raise a new model of urban intervention. A methodology was used proyectiva, to be able to study the elements in its context dynamic Relations, Creativity is interested by the evolutionary processes and the dynamic relations between the events and participation they are vital in the process of constructing a mobility alternatives and a few public spaces that they contribute. To promote the green axis as

\section{Introducción}

La expansión urbana de las ciudades intermedias en Colombia es un fenómeno urbano que se presenta a raíz de un crecimiento demográfico de las ciudades debido a la migración de campo a ciudad en busca de nuevas oportunidades económicas y culturales. Es así como el municipio de Sogamoso ha tenido un crecimiento demográfico y urbano que lo llevan a consolidarse como una ciudad intermedia que se desarrolla en medio de los desafíos de ciudades modernas de Latinoamérica que integrar el medio ambiente, las tecnologías y los derechos humanos como base fundamental para su expansión. Es por ello que desde el Plan de Ordenamiento Territorial (POT), la proyección del crecimiento urbano tiende a la ocupación y construcción vertical que concentre las ciudades y no alternative of economic and environmental development that from the solutions investigation for the social and environmental ecosystem inside the city for the rehabilitation of the green axis of "Rio Monquira" being the this one of our most important water sources, because the water bodies in a city are vital opposite to the reduction of $\mathrm{CO}$ and the improvement of the air.

Keywords: Climate change, green axis, mitigation, Rio Monquira

tenga una expansión urbana desmedida formando cinturones de miseria. En esta proyección se le hace una apuesta para aumentar el espacio público creando nuevos escenarios y rehabilita los existentes.

En la actualidad el espacio urbano de Sogamoso donde se proyecta la intervención es reducido, tiene problemas de seguridad, uso inadecuado de espacios públicos, zonas contaminadas ambientalmente, con problemas sociales y en especial de movilidad. La relación de los espacios públicos con la ciudad es inherente en la medida que se convierte en un derecho como de calidad y seguridad para el uso y disfrute de todos los grupos poblacionales, por ello es necesario la creación de nuevos espacios y la recuperación de lugares con deterioro, con la finalidad que la población cuente con parques, instalaciones deportivas y 
94

culturales, plazas u otros lugares que propicien el fortalecimiento de la vida en las ciudades.

\section{Metodología}

Al plantear un diagnóstico y levantamiento de información sobre el eje verde del Rio Monquira, es necesario tener diversas categorías que abarquen o midan desde los referentes conceptuales la información requerida para el desarrollo del trabajo, donde se tiene una parte teórica y una parte de investigación e indagación que en ambos casos se deben tener referentes relevantes $y$ concretos. Dicho trabajo se acompaña de una metodología proyectiva, que tiene como características:

a) Visión holística: estudia los elementos en su contexto

b) Relaciones dinámicas: se interesa en los procesos evolutivos y las relaciones dinámicas entre los eventos

c) Creatividad y participación: toma todos los actores del proceso

d) Actitud hacia el futuro y libertad para transformar los sucesos a partir de acciones voluntarias y dirigidas hacia ciertos fines.

Este tipo de investigación embarca nuestro trabajo, ya que está dirigida a la creación de un diagnostico real del Rio Monquira dejando un archivo históricoambiental del Rio que no existe en la actualidad ni lo ha desarrollado una entidad pública 0 privada. Esta metodología está acompañada de unas herramientas, cualitativas y cuantitativas que permiten tener un cruce de variables

Tabla 1. Categorías de análisis, variables e instrumentos

\begin{tabular}{|c|c|c|}
\hline CATEGORIAS & VARIABLES & INSTRUMENTOS \\
\hline Basuras & $\begin{array}{l}\text { Residencias } \\
\text { Comercio }\end{array}$ & $\begin{array}{l}\text { Observación: } \\
\text { Participante } \\
\text { NO participante }\end{array}$ \\
\hline Aguas & & Análisis Documental \\
\hline Empresas & \multirow[t]{4}{*}{$\begin{array}{l}\text { Industria } \\
\text { Comercio }\end{array}$} & Planos \\
\hline \multirow[t]{3}{*}{ Movilidad } & & Fotografías \\
\hline & & $\begin{array}{l}\text { Entrevistas Por } \\
\text { Sectores }\end{array}$ \\
\hline & & Medición Territorial \\
\hline Eje Ambiental & Pulmón Verde & Análisis Documental \\
\hline $\begin{array}{l}\text { Ciudades } \\
\text { Verdes }\end{array}$ & $\begin{array}{l}\text { Urbanismo } \\
\text { Sostenible }\end{array}$ & $\begin{array}{l}\text { Levantamientos De } \\
\text { Información }\end{array}$ \\
\hline Fitotectura & $\begin{array}{l}\text { Árboles } \\
\text { Nativos }\end{array}$ & Salida De Campo \\
\hline \multirow{2}{*}{$\begin{array}{l}\text { Cosmología } \\
\text { Muisca }\end{array}$} & \multirow{2}{*}{$\begin{array}{l}\text { Creencias } \\
\text { Ancestrales }\end{array}$} & Agua \\
\hline & & Vida \\
\hline $\begin{array}{l}\text { Espacio Público } \\
\text { Verde }\end{array}$ & $\begin{array}{l}\text { Espacio } \\
\text { Público } \\
\text { Colectivo }\end{array}$ & Análisis Documental \\
\hline $\begin{array}{l}\text { Movilidad } \\
\text { Alternativa }\end{array}$ & $\begin{array}{l}\text { Usos Del } \\
\text { Espacio } \\
\text { Publico }\end{array}$ & Estudio Documental \\
\hline $\begin{array}{l}\text { Espacios De } \\
\text { Movilidad } \\
\text { Alternativa }\end{array}$ & Ciclo vía & Talleres \\
\hline $\begin{array}{l}\text { Mitigación } \\
\text { Cambio } \\
\text { Climático }\end{array}$ & $\begin{array}{l}\text { Purificación } \\
\text { Del Aire }\end{array}$ & $\begin{array}{l}\text { Estudio De } \\
\text { Documentos } \\
\text { Climáticos }\end{array}$ \\
\hline $\begin{array}{l}\text { Espacio Urbano } \\
\text { Verde }\end{array}$ & $\begin{array}{l}\text { Parques } \\
\text { Urbanos }\end{array}$ & $\begin{array}{l}\text { Observación No } \\
\text { Participante }\end{array}$ \\
\hline \multirow[t]{2}{*}{$\begin{array}{l}\text { Cambio } \\
\text { Climático }\end{array}$} & \multirow[t]{2}{*}{$\begin{array}{l}\text { Transformación } \\
\text { De Ambiente }\end{array}$} & $\begin{array}{l}\text { Registro De } \\
\text { Información }\end{array}$ \\
\hline & & Grupo focal \\
\hline
\end{tabular}

\section{Resultados}


95

Los resultados que se han conseguido en este proceso de investigación, nos permite visualizar unas metas que tiene Sogamoso para ayudar a la mitigación del cambio climático. Nuestros resultados son basados en los avances investigativos y como lo mostramos en la siguiente tabla tenemos unos parciales a corto plazo y unos a largo plazo (tabla 2).

Tabla 2. Resultados esperados y parciales de la investigación

\begin{tabular}{|c|c|}
\hline Resultados parciales & Resultados Esperados \\
\hline $\begin{array}{l}\text { Medición de la } \\
\text { contaminación del } \\
\text { agua } \\
\text { Revisión de los } \\
\text { documentos existentes } \\
\text { de la historia del Rio } \\
\text { Identificación de los } \\
\text { principales } \\
\text { contaminantes del Rio }\end{array}$ & $\begin{array}{l}\text { Levantamiento } \\
\text { topográfico del rio } \\
\text { Análisis residual y de } \\
\text { aguas } \\
\text { Elaboración documento } \\
\text { de la historia del rio }\end{array}$ \\
\hline $\begin{array}{l}\text { Diagnóstico de estado } \\
\text { del Rio } \\
\text { Propuesta } \\
\text { mejoramiento frente a } \\
\text { la flora del Rio } \\
\text { Monquira }\end{array}$ & $\begin{array}{l}\text { Hacer un estudio de la } \\
\text { necesidad de un eje } \\
\text { verde dentro de la } \\
\text { ciudad } \\
\text { Tener una relación entre } \\
\text { la ubicación del Rio y la } \\
\text { cultura Muisca } \\
\text { Conocer los aportes } \\
\text { ambientales del actual } \\
\text { eje verde }\end{array}$ \\
\hline $\begin{array}{lr}\text { Adquisición de la } \\
\text { información ambiental } \\
\text { frente a la } \\
\text { contaminación del aire } \\
\text { Diagnóstico r del } \\
\text { espacio público actual } \\
\text { Determinación de los } \\
\text { usos del espacio } \\
\text { publico }\end{array}$ & $\begin{array}{l}\text { Resultados del flujo de } \\
\text { movilidad alrededor del } \\
\text { eje ambiental } \\
\text { Levantamiento de } \\
\text { información detallada de } \\
\text { espacios públicos } \\
\text { actuales y proyectados }\end{array}$ \\
\hline
\end{tabular}

\begin{tabular}{|c|c|}
\hline 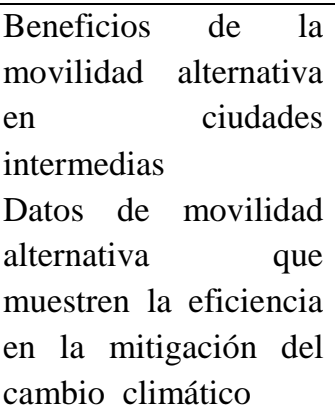 & $\begin{array}{l}\text { Registro de medición de } \\
\text { datos del crecimiento de } \\
\text { cause del Rio } \\
\text { Información detallada } \\
\text { ambiental del rio } \\
\text { Propuesta elaborada de } \\
\text { espacios para la } \\
\text { movilidad alternativa }\end{array}$ \\
\hline
\end{tabular}

Fuente: Elaboración propia

\section{Agradecimientos}

A la Universidad de Pamplona por permitirnos el espacio de contar y exponer nuestras ideas para poder crecer en ellas.

\section{Referencias Bibliogràficas}

Alcaldía Mayor de Bogotá (2000) Bogotá a escala humana Libro $\mathrm{N}^{\circ} 1$ Colección La Bogotá del tercer milenio: historia de una revolución urbana 1998 2000.

Díaz, R (2005) Lineamientos de diseño urbano en la gestión de proyectos de renovación en la ciudad de Bogotá, Universidad Nacional. Colombia. Arquitecto consultor de la Universidad Nacional. Arquitecto con énfasis en Urbanismo, Especialista en Proyecto Urbano, Magíster en Diseño Urbano, y candidato a Magíster en Economía Urbana con profundización en Política Pública, Consultor en Planificación, Gestión y Financiación del Desarrollo Urbano.

Londoño, M (2015). Centro Interactivo 2_10, Universidad Católica de Colombia, Facultad de diseño, programa de arquitectura, Bogotá. Colombia. Arquitecto de la universidad Católica 
de Colombia. Centro interactivo 2_10. Trabajo de Grado. Arquitecto de la Universidad Católica de Colombia. Facultad de Diseño. Programa de Arquitectura. Planificador urbano del Centro interactivo Bogotá, Colombia.
96

Recibido: Enero 10 de 2017

Aceptado:Enero 25 de 2018

Planeación municipal (2016) Plan de Ordenamiento Territorial Sogamoso, Sogamoso, Alcaldía Municipal. Recuperado de

Sogamoso Incluyente (2017) Alcaldía de Sogamoso, Sogamoso, Recuperado de: http://sogamosoboyaca.gov.co/index.shtml\#5

Vargas, Eileen (2015) "análisis de la problemática ambiental (recursos: aguaaire) que presenta el municipio de Sogamoso - Boyacá en el plan de ordenamiento territorial". Universidad Militar Nueva Granada. Bogotá D.C, Colombia.

Viviescas, Fernando (1997) Espacio público: Imaginación y Planeación Urbana en: La Calle: lo ajeno, lo público lo imaginado. Barrió Taller. Arquitecto y Urbanista: investigador, profesor universitario, teórico, crítico y consultor; es autor y coeditor de diversas publicaciones.

*Para citar este artículo: Sánchez Sierra M.F.; Villamarin Caro K.X.Environmental projection of the green axis of the Monquira River for the mitigation of pollution in Sogamoso Boyacá. Revista Bistua.2018.16(1):86-91.

+ Autor para el envió de correspondencia y la solicitud de las separatas: Sánchez Sierra M.F. Arquitectura Universidad de Boyacá. marfersanchez@uniboyaca.edu.co 